\title{
Continuous Murmur in a Young: Fistula from the Aortic Sinus of Valsalva to the Right Atrium
}

Ana Rita Santos ${ }^{1 *}$, Bruno Piçarra ${ }^{1}$, Carlos Cotrim ${ }^{2}$, Bruno Stuart ${ }^{2}$, Inês Cruz $^{2}$, Paula Fazendas ${ }^{2}$, Carlos Catarino ${ }^{2}$ and Hélder Pereira ${ }^{2}$ ${ }^{1}$ Serviço de Cardiologia, Hospital do Espirito Santo, EPE, Évora, Portugal

${ }^{2}$ Serviço de Cardiologia, Hospital Garcia de Orta, Almada, Portugal

\section{Case Report}

Sinus of Valsalva aneurysm (SVA) is a rare condition, with an incidence ranging from $0.09 \%-0.15 \%$ and comprises up to $3.5 \%$ of all congenital cardiac anomalies. SVA are frequently associated with other congenital heart disease, in particular the ventricular septal defects, but may also be acquired, arising as a complication of infective endocarditis, trauma, Behcet's disease, Marfan syndrome or syphilis [1,2].

Aneurysms may originate in the right coronary sinus (60\%), the noncoronary sinus (42\%), and, rarely, the left coronary sinus (10\%) [2].

SVA are usually diagnosed in the third or fourth decades of life as an incidental finding or after an acute rupture into an adjacent cardiac structure, being the right atrium the most frequently affected. When this rupture is present, a continuous cardiac murmur is an important physical sign [3]. About half of the aneurysms of SVA show a fistulous communication with a heart chamber or a great vessel. Thus, aneurysms of the right coronary sinus rupture preferentially into the right ventricle

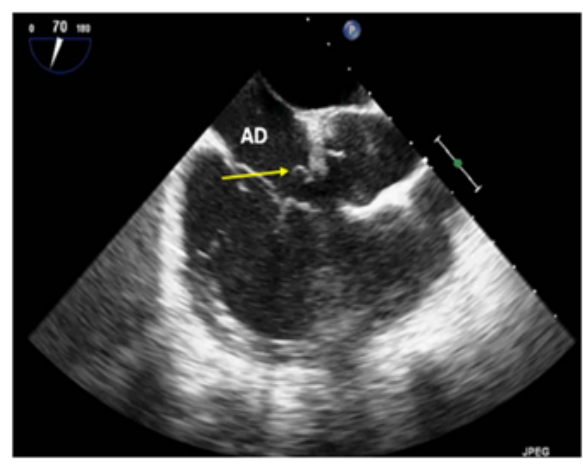

Figure 1: Transesophageal echocardiography - Plane with the aortic valve at $70^{\circ}$, observing an aneurysmal dilatation of the right coronary sinus of Valsalva (arrow) that is protruding into the right atrium (RA).

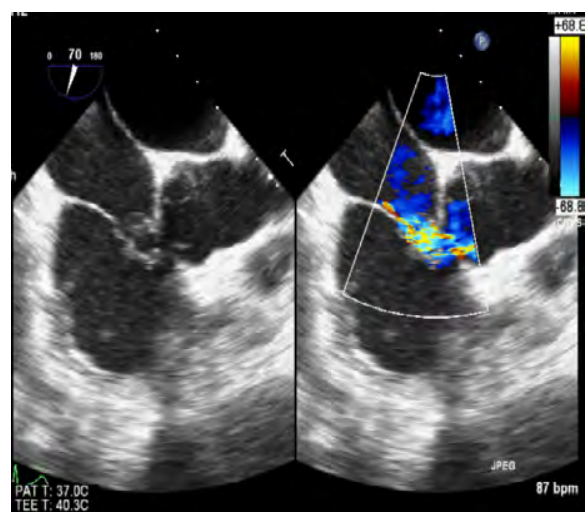

Figure 2: Transesophageal echocardiogram - Plane with aortic valve short axis at $40^{\circ}$. By Doppler color, it was verified a turbulent flow passage between the right coronary sinus to the right atrium.

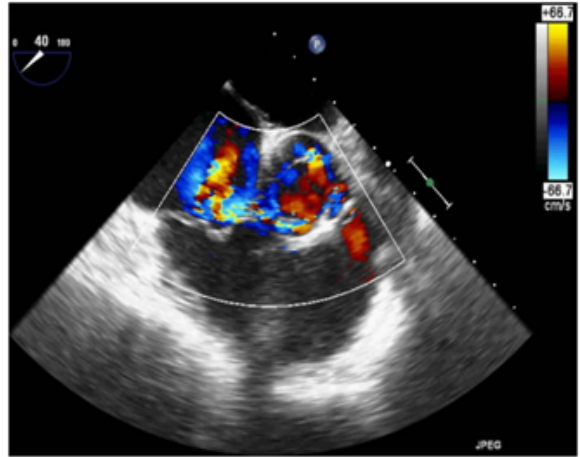

Figure 3: Images of transesophageal echocardiography, observing a continuity solution and a turbulent flow between the right sinus and the right atrium.

and aneurysms of non-coronary sinus rupture to the right atrium or to the right ventricle [4].

The management of an asymptomatic non ruptured aneurysm is not clear, however when the aneurysm is complicated by rupture the optimal management is surgical repair with an acceptably operative risk and good long term outcome $[5,6]$.

The authors described the case of a fistula between the right coronary sinus and the right atrium, whose diagnosis and anatomical characterization were established by transesophageal echocardiography.

A 30-year-old woman with no relevant personal or family history was referred to our hospital because of fatigue in moderate exercise and heart murmur. Her cardiovascular physical examination was significant for a "machine-type" continuous murmur of grade III/VI along the left and right paraesternal border. The transthoracic echocardiogram revealed the presence of possible aneurysm of the right coronary sinus.

To further characterize this pathology and exclusion of other associated cardiac anomalies, she rephrased a transesophageal echocardiogram which revealed the presence of a continuity solution between the right coronary sinus and the right atrium, compatible with a fistula between a small right coronary sinus aneurysms and the right atrium (Figures 1-3).

*Corresponding author: Dr. Ana Rita Santos, Serviço de Cardiologia, and Hospital do Espírito Santo, largo sr. pobreza 7000 - évora, Portugal, E-mail: anarita_asantos@hotmail.com

Received September 18, 2013; Accepted October 30, 2013; Published November 06, 2013

Citation: Santos AR, Piçarra B, Cotrim C, Stuart B, Cruz I, et al. (2013) Continuous Murmur in a Young: Fistula from the Aortic Sinus of Valsalva to the Right Atrium. J Cardiovasc Dis Diagn 1: 129. doi:10.4172/2329-9517.1000129

Copyright: ( $) 2013$ Santos AR, et al. This is an open-access article distributed under the terms of the Creative Commons Attribution License, which permits unrestricted use, distribution, and reproduction in any medium, provided the original author and source are credited. 
Citation: Santos AR, Piçarra B, Cotrim C, Stuart B, Cruz I, et al. (2013) Continuous Murmur in a Young: Fistula from the Aortic Sinus of Valsalva to the Right Atrium. J Cardiovasc Dis Diagn 1: 129. doi:10.4172/2329-9517.1000129

Page 2 of 2

The patient denied any history of infection or trauma. The patient was successfully submitted to surgical correction of the fistula and is currently asymptomatic.

\section{References}

1. Post MC, Braam RL, Groenemeijer BE, Nicastia D, Rensing BJ, et al. (2010) Rupture of right coronary sinus of Valsalva aneurysm into right ventricle. Neth Heart J 18: 209-211.

2. Galicia-Tornell MM, Marín-Solís B, Mercado-Astorga O, Espinoza-Anguiano S, Martínez-Martínez M, et al. (2009) Sinus of Valsalva aneurysm with rupture. Case report and literature review. Cir Cir 77: 441-445.
3. Gonzalez JB, Koul S, Sawardekar U, Bhat PQ, Kirshenbaum KJ, et al. (2008) Sinus of Valsalva Aneurysms: A unique case of Giant Aneurysms involving all 3 sinuses. Circulation 117: e308-e311.

4. Feldman DN, Gade CL, Roman MJ (2005) Ruptured Aneurysm of the Right Sinus of Valsalva Associated with a Ventricular Septal Defect and an Anomalous Coronary Artery. Tex Heart Inst J 32: 555-559.

5. Ibrahim KS, Waqfi NR, Jarrah MI (2013) Sinus of Valsalva Aneurysm with fistula to the right atrium presented as acute heart failure in a young man. Am $\mathrm{J}$ Case Rep 14: 398-400

6. Rescigno G, Matteucci ML, Capestro F, Pierri MD, Bucari S, et al. (2010) A Ruptured right sinus of Valsalva diverticled aneurysm: echocardiographic diagnosis and surgical repair. Minerva Chir 65: 123-126. 